PROCEEDINGS OF THE

AMERICAN MATHEMATICAL SOCIETY

Volume 136, Number 6, June 2008, Pages 2225-2233

S 0002-9939(08)09149-1

Article electronically published on February 14, 2008

\title{
ASYMPTOTIC CONES AND ASSOUAD-NAGATA DIMENSION
}

\author{
J. DYDAK AND J. HIGES
}

(Communicated by Alexander N. Dranishnikov)

\begin{abstract}
We prove that the dimension of any asymptotic cone over a metric space $(X, \rho)$ does not exceed the asymptotic Assouad-Nagata dimension $\operatorname{asdim}_{A N}(X)$ of $X$. This improves a result of Dranishnikov and Smith (2007), who showed $\operatorname{dim}(Y) \leq \operatorname{asdim}_{A N}(X)$ for all separable subsets $Y$ of special asymptotic cones $\operatorname{Cone}_{\omega}(X)$, where $\omega$ is an exponential ultrafilter on natural numbers.

We also show that the Assouad-Nagata dimension of the discrete Heisenberg group equals its asymptotic dimension.
\end{abstract}

\section{INTRODUCTION}

Given a metric space $\left(X, \rho_{X}\right)$ and given a sequence $d=\left\{d_{n}\right\}_{n=1}^{\infty}$ of real numbers diverging to infinity, one constructs an asymptotic cone Cone $\omega(X, c, d)$ for every non-principal ultrafilter $\omega$ in natural numbers $\mathbf{N}$ as follows:

First one considers the pseudo-metric space of all functions $x: N \rightarrow X$ such that $\left\{\frac{\rho_{X}(x(n), c(n))}{d_{n}}\right\}$ is bounded for a given function $c: N \rightarrow X$ (that function serves as the base point of the asymptotic cone). The distance from $x$ to $y$ is the $\omega$-limit $\lim _{\omega} \frac{\rho_{X}(x(n), y(n))}{d_{n}}$ of $\left\{\frac{\rho_{X}(x(n), y(n))}{d_{n}}\right\}$ (see [2] or [13] for details). By identifying $x$ and $y$ whose distance is 0 one gets a metric space Cone $_{\omega}(X, c, d)$.

One can generalize the definition of asymptotic cones for non-principal ultrafilters $\omega$ over any infinite set $I$ (see [18]). In that case one requires that $d=\left\{d_{i}\right\}_{i \in I}$ has the property that the $\omega$-limit of $\left\{\frac{1}{d_{i}}\right\}_{i \in I}$ is 0 . As all our proofs are valid for that case, we will work in that generality.

An important problem in geometric group theory is relating properties of finitely generated groups $G$ to topological properties of their asymptotic cones (i.e., asymptotic cones of their Cayley graphs):

- A finitely generated group is virtually Abelian if and only if its asymptotic cones are isometric to the space $\mathbf{R}^{n}[14,[22$.

- A finitely generated group is virtually nilpotent if and only if its asymptotic cones are locally compact [14, 24], [12].

Received by the editors October 20, 2006.

2000 Mathematics Subject Classification. Primary 54F45; Secondary 55M10, 54C65.

Key words and phrases. Assouad-Nagata dimension, asymptotic dimension, asymptotic cones, covering dimension.

The first author was partially supported by the Center for Advanced Studies in Mathematics at Ben Gurion University of the Negev (Beer-Sheva, Israel).

The second author is supported by Grant AP2004-2494 from the Ministerio de Educación y Ciencia, Spain.

(C) 2008 American Mathematical Society Reverts to public domain 28 years from publication 
- A finitely generated group is hyperbolic if and only if its asymptotic cones are $\mathbf{R}$-trees [15].

- A finitely generated group $G$ is relatively hyperbolic with respect to the finitely generated subgroups $H_{1}, \ldots, H_{n}$ if and only if every asymptotic cone of $G$ is tree-graded with respect to $\omega$-limits of sequences of cosets of the subgroups $H_{i}[13$.

It would be of interest to detect the property of a finitely generated group $G$ equivalent to finite-dimensionality of its asymptotic cones.

The first time the dimension of asymptotic cones was discussed occurred in [14, where M. Gromov proved finite-dimensionality of asymptotic cones of a finitely generated group of polynomial growth by demonstrating that their Hausdorff dimension is finite.

J. Burillo [8] showed 1-dimensionality of asymptotic cones of Baumslag-Solitar groups and used it to demonstrate that the fundamental group of those cones is not free and uncountable.

More recently, J. Behrstock and Y. Minsky [3] proved the following:

Theorem 1.1 (Dimension Theorem of Behrstock-Minsky). The maximal topological dimension of a locally compact subset of the asymptotic cone of a mapping class group is equal to the maximal rank of an abelian subgroup.

The aim of this note is to relate asymptotic Assouad-Nagata dimension of a finitely generated group $G$ to the dimension of its asymptotic cones. More generally, we prove $\operatorname{dim}\left(\operatorname{Cone}_{\omega}(X, c, d)\right) \leq \operatorname{asdim}_{A N}(X)$ for all metric spaces $(X, \rho)$ and all non-principal ultrafilters $\omega$. Its direct predecessor is the following result from [10]:

Theorem 1.2 (Dranishnikov-Smith). $\operatorname{dim}(Y) \leq \operatorname{asdim}_{A N}(X)$ for every separable subset $Y \subset \mathrm{Cone}_{\omega}(X)$ if $X$ is a proper metric space and $\omega$ is an exponential ultrafilter in $\mathbf{N}$.

Notice that the asymptotic cones in Theorem 1.2 are based on the sequence $d_{n}=n$.

The way Theorem 1.2 was proved in 10 was by constructing an injective map Cone $_{\omega}(X) \backslash\left[x_{0}\right] \rightarrow \nu_{L}(X)$, where $\nu_{L}(X)$ is the sublinear Higson corona of $X$ (see Theorem 5.1 of [10]). Since $\operatorname{asdim}_{A N}(X) \geq \operatorname{dim}\left(\nu_{L}(X)\right)$ for every proper metric space $(X, d)$ (see Theorem 3.10 in [10]), Theorem 1.2 follows.

It is not clear if the methods of this paper allow us to improve Theorem 1.1 and show that the dimension of asymptotic cones of mapping class groups equals the maximal rank of an abelian subgroup. We learned from Jason Behrstock that in order to remove the 'locally compact' hypothesis in Theorem 1.1 all one needs to show is that the dimension of asymptotic cones of mapping class groups is finite. That leads to the question of Assouad-Nagata dimension of mapping class groups being finite. That question remains open. Even the finiteness of the asymptotic dimension of mapping class groups remains open (except in genus less than 3) (see [5] for an overview of asymptotic dimension).

We are grateful to Jason Behrstock and Kolya Brodskiy for valuable comments.

\section{Assouad-Nagata Dimension}

A metric space $(X, \rho)$ is of Assouad-Nagata dimension (see [19] and [1]) at most $n$ (notation: $\operatorname{dim}_{A N}(X) \leq n$ ) if there is a constant $c>0$ such that for any $r>0$, 
there is a cover $\mathcal{U}_{r}$ of $X$ whose elements have diameter at most $c \cdot r$ and every $r$-ball $B(x, r)$ intersects at most $n+1$ elements of $\mathcal{U}_{r}$. Equivalently (see [7]), there is a constant $K>0$ such that for any $r>0, X$ can be expressed as $X=X_{0} \cup \ldots \cup X_{n}$ so that all $r$-components of $X_{i}$ are $K \cdot r$-bounded (two points are in the same $r$ component of a metric space if they can be connected by a finite chain of points so that each link is of size less than $r$ ).

If the existence of $\mathcal{U}_{r}$ is guaranteed only for sufficiently large $r$, then we say the asymptotic Assouad-Nagata dimension of $X$ is at most $n$ (notation: $\operatorname{asdim}_{A N}(X) \leq$ $n)$.

For connections of Assouad-Nagata dimension to Lipschitz extensions, see [17] and 6 .

Of basic importance to us is the following result:

Theorem 2.1 (Lang-Schlichenmaier [17]). If $f:\left(X, \rho_{X}\right) \rightarrow\left(Y, \rho_{Y}\right)$ is a quasisymmetric embedding of metric spaces, then $\operatorname{dim}_{A N}(X) \leq \operatorname{dim}_{A N}(Y)$.

We will apply Theorem 2.1 in the case of bi-Lipschitz homeomorphisms and snowflaking. Recall that a snowflaked version of a metric space $(X, \rho)$ is the space $\left(X, \rho^{p}\right)$ for some $0<p<1$ (see [16], p. 98).

$(X, \rho)$ has the $n$-dimensional Nagata property if for every configuration $y_{1}, \ldots$, $y_{n+2}$ of $n+2$ points in $X$ the conditions $\rho\left(y_{i}, B(x, r / 2)\right)<r$ for some $x \in X$ and all $i \leq n+2$ imply the existence of $i \neq j$ such that $\rho\left(y_{i}, y_{j}\right)<r$.

Theorem 2.2. Let $\left(X, \rho_{X}\right)$ be a metric space and $n \geq 0 . \operatorname{dim}_{A N}(X) \leq n$ if and only if $\left(X, \rho_{X}\right)$ is bi-Lipschitz homeomorphic to a metric space $\left(Y, \rho_{Y}\right)$ such that some snowflaked version of $\left(Y, \rho_{Y}\right)$ has the $n$-dimensional Nagata property.

Proof. In one direction it follows from Theorem 2.1 and the fact that $\operatorname{dim}_{A N}(Y) \leq n$ if $Y$ has the $n$-dimensional Nagata property. Indeed, if $r>0$ and $A$ is a maximal subset of $Y$ with respect to the property $\rho(x, y) \geq r$ for all $x \neq y \in A$, then the family of balls $\mathcal{U}_{r}=\{B(y, r)\}_{y \in A}$ covers $Y$ and any ball $B(x, r / 2)$ intersects at most $n+1$ elements of $\mathcal{U}_{r}$ (see the proof of Theorem 4.2 in [11] along the same lines).

The other direction is proved in Proposition 2.2 of [1].

\section{MAPS With METRICALLY PARALLEL FiBERS}

J. Burillo [8] defined a very interesting class of maps, maps with metrically parallel fibers. Recall $f:\left(X, \rho_{X}\right) \rightarrow\left(Y, \rho_{Y}\right)$ is a map with metrically parallel fibers if for every $x \in X$ and $y \in f(X)$ there is $x^{\prime} \in f^{-1}(y)$ such that $\rho_{X}\left(x, x^{\prime}\right)=$ $\rho_{Y}\left(f(x), f\left(x^{\prime}\right)\right)=\rho_{Y}(f(x), y)$ (see Definition 6 in $[$ ] ). It seems maps with metrically parallel fibers provide a natural ground for applying Hurewicz type theorems from [7] (see the proof of Theorem 3.4).

\section{Examples of maps with metrically parallel fibers.}

(1) The projection $X \times Y \rightarrow Y$ if the metric on $X \times Y$ is either Euclidean or the sum of corresponding metrics on $X$ and $Y$.

(2) Im: $\mathbf{H}^{2} \rightarrow \mathbf{R}$, where $\mathbf{H}^{2}$ is the hyperbolic plane in the upper half plane model (see 8$]$ ). Notice $\mathbf{R}$ is identified with $(0, \infty)$ equipped with the metric $d(x, y)=|\ln (x)-\ln (y)|$. 
We will weaken the condition of the existence of an $x^{\prime}$ such that $\rho_{X}\left(x, x^{\prime}\right)=$ $\rho_{Y}\left(f(x), f\left(x^{\prime}\right)\right)=\rho_{Y}(f(x), y)$ to simply $\rho_{X}\left(x, f^{-1}(y)\right) \leq \rho_{Y}(f(x), y)$ for all $x \in X$ and $y \in f(X)$. One reason for the change is that the following natural functions easily satisfy the new condition.

(1) The function $x \rightarrow d\left(x, x_{0}\right)$ from any $\mathbf{R}$-tree $T$ to $\mathbf{R}_{+}$, where $x_{0}$ is a base point of $T$. We assume every point of $T$ lies inside an infinite geodesic.

(2) Any epimorphism $f:\left(G, d_{G}\right) \rightarrow\left(H, d_{H}\right)$ of finitely generated groups equipped with word metrics induced by finite sets of generators $S$ in $G$ and $f(S)$ in $H$.

The second reason is the following.

Proposition 3.1. If $f:\left(X, \rho_{X}\right) \rightarrow\left(Y, \rho_{Y}\right)$ is a Lipschitz map, then the following conditions are equivalent:

a. The natural function $f^{-1}(y) \rightarrow y$ establishes a bi-Lipschitz equivalence between $f(X)$ and the space of fibers of $f$ equipped with the Hausdorff distance.

b. There is $\mu>0$ such that $\rho_{X}\left(x, f^{-1}(y)\right) \leq \mu \cdot \rho_{Y}(f(x), y)$ for all $x \in X$ and $y \in f(X)$.

Proof. a) $\Longrightarrow$ b). Recall the Hausdorff distance $d_{H}(A, B)$ between two subsets $A$ and $B$ of $X$ is the supremum of $\rho_{X}(a, B)$ and $\rho_{X}(b, A)$ over all $a \in A$ and $b \in B$. A surjective map $g: S \rightarrow T$ of metric spaces is a bi-Lipschitz equivalence if there are constants $\lambda, \mu>0$ such that $\lambda \cdot d_{T}(g(a), g(b)) \leq d_{S}(a, b) \leq$ $\mu \cdot d_{T}(g(a), g(b))$ for all $a, b \in S$. Thus b) follows easily from a) as $\rho_{X}\left(x, f^{-1}(y)\right) \leq$ $d_{H}\left(f^{-1}(f(x)), f^{-1}(y)\right) \leq \mu \cdot \rho(f(x), y)$ for all $x \in X$ and $y \in f(X)$.

b) $\Longrightarrow$ a). Assume $f$ is $\lambda$-Lipschitz (which means $\rho(f(x), f(y)) \leq \lambda \cdot \rho_{X}(x, y)$ for all $x, y \in X)$ and $y_{1}, y_{2} \in f(X)$. Since $\rho_{X}\left(x, f^{-1}\left(y_{2}\right)\right) \leq \mu \cdot \rho\left(y_{1}, y_{2}\right)$ for all $x \in f^{-1}\left(y_{1}\right)$ and $\rho_{X}\left(x, f^{-1}\left(y_{1}\right)\right) \leq \mu \cdot \rho\left(y_{1}, y_{2}\right)$ for all $x \in f^{-1}\left(y_{2}\right)$, we get $d_{H}\left(f^{-1}\left(y_{1}\right), f^{-1}\left(y_{2}\right)\right) \leq \mu \cdot \rho_{Y}\left(y_{1}, y_{2}\right)$. Similarly, as $f$ is $\lambda$-Lipschitz, one has $\lambda$. $\rho_{X}\left(x, f^{-1}\left(y_{2}\right)\right) \geq \rho_{Y}\left(y_{1}, y_{2}\right)$ for all $x \in f^{-1}\left(y_{1}\right)$ resulting in $\frac{\rho_{Y}\left(y_{1}, y_{2}\right)}{\lambda} \leq$ $d_{H}\left(f^{-1}\left(y_{1}\right), f^{-1}\left(y_{2}\right)\right)$ for all $y_{1}, y_{2} \in f(X)$.

Proposition 3.1 provides a global explanation for the term 'metrically parallel fibers'. Also, after rescaling $Y$ one can simply achieve $\mu=1$ in Proposition 3.1, so that is going to be our assumption.

It was suggested to us by K. Brodskiy that maps as in Proposition 3.1(b) ought to be called metrically open due to the following:

Proposition 3.2 (K. Brodskiy). Let $f: X \rightarrow Y$ be a surjective map of metric spaces. The following properties are equivalent:

1. There exists $\mu>0$ such that for any $x \in X$ and $y \in Y$,

$$
d_{X}\left(x, f^{-1}(y)\right) \leq \mu d_{Y}(f(x), y) .
$$

2. There exists $\lambda>0$ such that for any $x \in X$ and any $R>0$,

$$
B(f(x), \lambda R) \subset f(B(x, R)) .
$$

Proof. In both directions assume the relation $\mu=1 / \lambda$.

$1) \Longrightarrow 2$ ). If $d_{Y}(f(x), f(z))<\lambda R$ and $f(z)=y$, then $d_{X}\left(x, f^{-1}(y)\right)<R$, so there is $t \in f^{-1}(y)$ satisfying $d_{X}(x, t)<R$. Thus $y=f(t) \in f(B(x, R))$.

$2) \Longrightarrow 1)$. Suppose $d_{Y}(f(x), y)<\lambda R$. That means $y \in B(f(x), \lambda R)$ and there is $z \in B(x, R)$ so that $y=f(z)$. Thus $d_{X}\left(x, f^{-1}(y)\right)<R$, which proves $d_{X}\left(x, f^{-1}(y)\right) \leq \mu d_{Y}(f(x), y)$. 
J. Burillo proved in 8 (Theorem 16) that $\operatorname{dim}(X) \leq \operatorname{dim}(Y)$ if there is a map $f: X \rightarrow Y$ with parallel fibers such that $f^{-1}(y)$ is an ultrametric space for all $y \in Y$. Recall $(A, \rho)$ is ultrametric if $\rho(x, z) \leq \max (\rho(x, y), \rho(y, z))$ for all triples $x, y, z$ in $A$. Our analog of that result for Assouad-Nagata dimension has a much simpler proof (if Lipschitz maps are involved):

Theorem 3.3. Suppose $f:\left(X, \rho_{X}\right) \rightarrow\left(Y, \rho_{Y}\right)$ is a Lipschitz map with ultrametric fibers. If

$$
\rho_{X}\left(x, f^{-1}(y)\right) \leq \rho_{Y}(f(x), y)
$$

for all $x \in X$ and $y \in f(X)$, then $\operatorname{dim}_{A N}(X) \leq \operatorname{dim}_{A N}(Y)$.

Proof. Let $f$ be $\lambda$-Lipschitz. Let us show that if $B \subset Y$ and all $\lambda \cdot r$-components of $B$ are $K$-bounded, then all $r$-components of $f^{-1}(B)$ are $(4 K+r)$-bounded. Indeed, if $x_{1}, \ldots, x_{n}$ is a chain of points such that $f\left(x_{i}\right) \in B$ and $\rho_{X}\left(x_{i}, x_{i+1}\right)<r$ for all $i<n$, then the $f\left(x_{i}\right)$ belong to the same $\lambda \cdot r$-component of $B$. Hence there is a fiber $F \subset f^{-1}(B)$ such that $\rho_{X}\left(x_{i}, F\right)<K$ for all $i \leq n$ and we can pick $y_{i} \in F$ satisfying $\rho_{X}\left(x_{i}, y_{i}\right)<K$ for all $i \leq n$. Thus all $y_{i}$ belong to the same $(2 K+r)$ component of $F$. As $F$ is ultrametric, that component is of size less than $2 K+r$. Therefore $\rho_{X}\left(x_{1}, x_{n}\right) \leq 2 K+\rho_{X}\left(y_{1}, y_{n}\right)<2 K+2 K+r=4 K+r$.

Suppose $\operatorname{dim}_{A N}(Y)=m<\infty$ (the case $\operatorname{dim}_{A N}(Y)=\infty$ is trivial) and let $c>0$ be the corresponding constant for $Y$. Given $r>0$ pick a cover $\left\{Y_{1}, \ldots, Y_{m+1}\right\}$ of $Y$ such that any $\lambda \cdot r$-component of each $Y_{i}$ has diameter less than $c \cdot \lambda \cdot r$. Now $r$-components of each set $f^{-1}\left(Y_{i}\right)$ are bounded by $(4 c \lambda+1) \cdot r$, which proves $\operatorname{dim}_{A N}(X) \leq m$.

Notice Theorem 3.3 implies the well-known fact (see [17]) $\operatorname{dim}_{A N}(T) \leq 1$ for any R-tree $T$ using $f: T \rightarrow \mathbf{R}_{+}, f(x)=d\left(x, x_{0}\right)$.

Bell and Dranishnikov [4 introduced the concept of asdim $\left\{A_{s}\right\}_{s \in S} \leq n$ uniformly with respect to $s \in S$. The analogous concept of $\operatorname{dim}_{A N}\left(A_{s}\right) \leq n$ uniformly with respect to $s \in S$ means that the constant $c$ in the definition of Assouad-Nagata dimension can be chosen the same for all $A_{s}, s \in S$. Geometrically, the most common occurrence is when all $A_{s}$ are isometric.

Here is a generalization of Theorem 3.3 .

Theorem 3.4. Suppose that $f:\left(X, \rho_{X}\right) \rightarrow\left(Y, \rho_{Y}\right)$ satisfies $\rho_{X}\left(x, f^{-1}(y)\right) \leq$ $\rho_{Y}(f(x), y)$ for all $x \in X$ and $y \in Y$. If $f$ is Lipschitz and

$$
\operatorname{dim}_{A N}\left(f^{-1}(y)\right) \leq k
$$

uniformly with respect to $y \in f(X)$, then $\operatorname{dim}_{A N}(X) \leq k+\operatorname{dim}_{A N}(Y)$.

Proof. The proof consists of applying Theorem 7.1 in [7. That result says that $\operatorname{dim}_{A N}(X) \leq \operatorname{dim}_{A N}(f)+\operatorname{dim}_{A N}(Y)$ if $f$ is Lipschitz. We need to show $\operatorname{dim}_{A N}(f) \leq$ $k$, which means the existence of constants $a$ and $b$ such that for any $r_{X}, R_{Y}>0$ and any subset $A$ of $X$ so that $f(A)$ is $R_{Y}$-bounded, $A$ can be expressed as $A=$ $A_{0} \cup \ldots \cup A_{k}$ with $r_{X}$-components of each $A_{i}$ being $\left(a \cdot r_{X}+b \cdot R_{Y}\right)$-bounded.

Assume $f$ is $\lambda$-Lipschitz. Pick $y \in f(A)$ and put $F=f^{-1}(y)$. Cover $F$ with sets $F_{0}, \ldots, F_{k}$ such that $\left(2 R_{Y}+r_{X}\right)$-components of $F_{i}$ are $c \cdot\left(2 R_{Y}+r_{X}\right)$-bounded. Look at $A_{i}=B\left(F_{i}, R_{Y}\right)$ and notice its $r_{X}$-components are $\left(c\left(2 R_{Y}+r_{X}\right)+2 R_{Y}\right)$ bounded. As the $A_{i}$ cover $A$ (use $\rho_{X}(x, F) \leq \rho_{Y}(f(x), f(F))<R_{Y}$ for all $x \in A$ ), $a=c$ and $b=2 c+2$ work. 


\section{Dimension of ASYMPtotic CONES}

Proposition 4.1. Let $\left(X, \rho_{X}\right)$ be a metric space and $n \geq 0$. If $\left(X, \rho_{X}\right)$ has the $n$-dimensional Nagata property, then every asymptotic cone over $X$ has the $n$-dimensional Nagata property.

Proof. Let's rephrase the $n$-dimensional Nagata property as follows: given $r>0$ and points $y_{i}, z_{i}, i \leq n+2$, such that $\rho_{X}\left(y_{i}, z_{i}\right)<r$ and $\rho_{X}\left(x, z_{i}\right)<r / 2$ for some $x \in X$ and all $i \leq n+2$, there is $i \neq j$ such that $\rho_{X}\left(y_{i}, y_{j}\right)<r$.

Suppose $\left(Y, \rho_{Y}\right)=\operatorname{Cone}_{\omega}(X, c, d)$ does not have the $n$-dimensional Nagata property and there exist points $x \in Y, y_{i}, z_{i} \in Y$ for $i \leq n+2$ such that for some $r>\epsilon>0$ the following inequalities hold:

a. $\rho_{Y}\left(y_{i}, z_{i}\right)<r-\epsilon$ and $\rho_{Y}\left(x, z_{i}\right)<r / 2-\epsilon / 2$ for all $i \leq n+2$,

b. $\rho_{Y}\left(y_{i}, y_{j}\right) \geq r$ for all $i \neq j$.

For each $i$ pick $P_{i} \in \omega$ such that $\rho_{X}\left(y_{i}(n), z_{i}(n)\right)<(r-\epsilon) \cdot d_{n}$ and $\rho_{X}\left(z_{i}(n), x(n)\right)<$ $(r / 2-\epsilon / 2) \cdot d_{n}$ for all $n \in P_{i}$. Similarly, for all $i \neq j$, pick $P_{i j} \in \omega$ such that $\rho_{X}\left(y_{i}(n), y_{j}(n)\right) \geq(r-\epsilon) \cdot d_{n}$ for all $n \in P_{i j}$. Let $P$ be the intersection of all $P_{i}$ and all $P_{i j}$. Since $P \neq \emptyset$, we arrive at a contradiction: $\left(X, \rho_{X}\right)$ cannot have the $n$-dimensional Nagata property.

Corollary 4.2. If $\left(X, \rho_{X}\right)$ is ultrametric, then every asymptotic cone Cone ${ }_{\omega}(X, c, d)$ is ultrametric.

Proof. It follows from the well-known fact that being ultrametric is the same as having the 0-dimensional Nagata property (easy exercise left to the reader).

Corollary 4.3. For any metric space $\left(X, \rho_{X}\right)$,

$$
\operatorname{dim}\left(\operatorname{Cone}_{\omega}(X, c, d)\right) \leq \operatorname{dim}_{A N}\left(\operatorname{Cone}_{\omega}(X, c, d)\right) \leq \operatorname{asdim}_{A N}\left(X, \rho_{X}\right) .
$$

Proof. Suppose $\operatorname{asdim}_{A N}\left(X, \rho_{X}\right)=n$. Notice Cone $\operatorname{Con}_{\omega}\left(X, \rho_{X}, d\right)$ is isometric to Cone $_{\omega}\left(X, \rho_{2}, d\right)$, where $\rho_{2}(x, y)=\rho_{X}(x, y)$ if $\rho_{X}(x, y) \geq 1$ and $\rho_{2}(x, y)=1$ if $0<\rho_{X}(x, y)<1$. Also, $\operatorname{dim}_{A N}\left(X, \rho_{2}\right)=n$ as $\operatorname{asdim}_{A N}\left(X, \rho_{2}\right)=n$ and the microscopic Assouad-Nagata dimension of $\left(X, \rho_{2}\right)$ is 0 (see [6]). By Theorem 2.2 there is a metric space $\left(Y, \rho_{Y}\right)$ bi-Lipschitz equivalent to $\left(X, \rho_{2}\right)$ such that $\left(Y, \rho_{Y}^{p}\right)$ has the $n$-dimensional Nagata property for some $0<p<1$. Therefore $\operatorname{Cone}_{\omega}\left(Y, \rho_{Y}, d\right)$ is bi-Lipschitz equivalent to $\operatorname{Cone}_{\omega}\left(X, \rho_{X}, d\right)$. Since the snowflaked version $\operatorname{Cone}_{\omega}\left(Y, \rho_{Y}^{p}, d^{p}\right)$ of $\operatorname{Cone}_{\omega}\left(Y, \rho_{Y}, d\right)$ has the $n$-dimensional Nagata property by Proposition 4.1. we conclude

$$
\operatorname{dim}_{A N}\left(\text { Cone }_{\omega}\left(X, \rho_{X}, d\right)\right) \leq n .
$$

As $\operatorname{dim}(Y) \leq \operatorname{dim}_{A N}(Y)$ for all metric spaces (see [17], Theorem 2.2), the proof is completed.

J. Burillo [8] showed that asymptotic cones of Baumslag-Solitar groups $B S_{p, q}$ (groups with generators $t, x$ and the sole relation $t^{-1} x^{p} t=x^{q}$ where $p \neq q$ ) have dimension 1 by displaying a map $f$ from the Cayley graph of $B S_{p, q}$ to an $\mathbf{R}$ tree $T$ with metrically parallel fibers such that the fibers of the induced maps on asymptotic cones are ultrametric. In view of Corollary 4.3 and Theorem 3.3 one can improve his result by stating that asymptotic cones of $B S_{p, q}$ are of Assouad-Nagata dimension 1 . 
Question 4.4. Is there a finitely generated group whose asymptotic cones have bounded Assouad-Nagata dimension but the group itself has infinite asymptotic Assouad-Nagata dimension?

Question 4.5. Is there $(X, \rho)$ of positive asymptotic Assouad-Nagata dimension whose asymptotic cones are of Assouad-Nagata dimension at most 0 ?

\section{Assouad-Nagata dimension of the discrete Heisenberg group}

We do not know of a finitely presented group $G$ of finite asymptotic dimension whose asymptotic Assouad-Nagata dimension is larger but finite. Piotr Nowak 20. identified finitely generated groups $G_{n}$ of asymptotic dimension $n \geq 1$ and $\operatorname{asdim}_{A N}\left(G_{n}\right)=\infty$. A.N. Dranishnikov communicated to us that there was a suggestion by John Roe that perhaps the Heisenberg group (see [23] for its discussion) may provide an example where the dimensions differ. However, that is not the case 1

Proposition 5.1. The asymptotic dimension and Assouad-Nagata dimension of the discrete Heisenberg group $\mathrm{H}_{3}(\mathbf{Z})$ both equal 3 .

Proof. Recall (see [15], p. 51) that the continuous Heisenberg group $H_{3}(\mathbf{R})$ is the only non-Abelian nilpotent three-dimensional group. It contains, as a cocompact lattice, the discrete Heisenberg group $H_{3}(\mathbf{Z})$ generated by $a, b$, and $c$ with the relations $c=[a, b],[a, c]=1$, and $[b, c]=1$. Since $G=H_{3}(\mathbf{Z})$ is of Hirsch length 3, its asymptotic dimension is 3 (see Theorem 3.5 in $[9]$ ). To observe $\operatorname{asdim}_{A N}(G) \leq 3$ consider the exact sequence $1 \rightarrow \mathbf{Z} \rightarrow G \rightarrow G / \mathbf{Z} \rightarrow 1$, where $\mathbf{Z}$ is generated by $c$. As $G / \mathbf{Z}=\mathbf{Z} \oplus \mathbf{Z}$, it suffices to show $(\mathbf{Z}, d)$ is quasi-symmetrically equivalent to $\mathbf{Z}$ equipped with the regular metric, where $d$ is a metric on $\mathbf{Z}$ induced from a word metric on $G$. Indeed, in view of Theorem 2.1 one gets $\operatorname{asdim}_{A N}(\mathbf{Z}, d)=1$, and applying Theorem 3.4 one derives $3=\operatorname{asdim}(G) \leq \operatorname{asdim}_{A N}(G) \leq 3$.

As suggested in [15] on p. 52 the metric $d$ is quasi-isometric to the square root of the regular metric on $\mathbf{Z}$ (the equality $\left[a^{n}, b^{n}\right]=c^{n^{2}}$ is cited). Since that statement is not totally obvious, let us provide a detailed exposition of the following:

Claim 1. Let $K>16$ be sufficiently large so that $4(n+1)+K \cdot \sqrt{n} \leq K \cdot n$ for all $n \geq 2$ (equivalently, $K \geq \frac{4(n+1)}{n-\sqrt{n}}$ for all $n \geq 2$ ). For every integer $k$ there is a word $w$ in $a$ and $b$ (including inverses) such that $w=c^{k}$ in $G$ and $l(w) \leq K \sqrt{|k|}$, where $l(w)$ is the length of $w$.

Proof of Claim 1. We will use equality $\left[a^{u}, b^{v}\right]=c^{u \cdot v}$, which we leave to the reader to prove. It suffices to consider $k$ positive and the proof is by induction on $k$. If $k=n^{2}$ for some natural $n$, then we put $w=\left[a^{n}, b^{n}\right]$ whose length is $4 \cdot n$. For $k=2$ one takes $w=[a, b]^{2}$. Similarly, $k=3$ is handled. If there is natural $n \geq 2$ such that $n^{2}<k \leq n^{2}+n$, then we pick a word $w^{\prime}$ for $k^{\prime}=k-n^{2}$ and we put $w=\left[a^{n}, b^{n}\right] \cdot w^{\prime}$. Notice $l(w) \leq 4 n+l\left(w^{\prime}\right) \leq 4 n+K \sqrt{k-n^{2}} \leq K n \leq K \sqrt{k}$. Otherwise there is $n \geq 2$ such that $(n+1)^{2}-n \leq k<(n+1)^{2}$, in which case we put $k^{\prime}=k-(n+1)^{2}$ and $w=\left[a^{n+1}, b^{n+1}\right] \cdot w^{\prime}$, where $w^{\prime}=c^{k^{\prime}}$ and $l\left(w^{\prime}\right) \leq K \sqrt{n}$.

Claim 2. If $w$ is a word in $a$ and $b$ (including inverses) such that $w=c^{k}$ in $G$ for some $k$, then $2 \sqrt{|k|} \leq l(w)$.

\footnotetext{
${ }^{1}$ When the paper was written we learned from Dranishnikov that he and P. Nowak proved this fact independently.
} 
Proof of Claim 2. By contradiction. Find $w$ of minimal length such that $w=c^{k}$ and $l(w)<2 \sqrt{|k|}$. Among such words (minimizing $l(w)$ ) choose a word maximizing $|k|$ (that maximum cannot be infinity as there are only finitely many words of a given length). Notice $l(w)>4$. Since $c$ is in the center of $G$, equality $x \cdot y=c^{k}$ implies $y \cdot x=c^{k}$. Therefore (via a cyclic permutation) we may assume $w=a^{m(1)}$. $b^{n(1)} \cdot \ldots \cdot a^{m(s)} \cdot b^{n(s)}$, where all exponents are non-zero and $l(w)=\sum(|m(i)|+|n(i)|)$. Notice $\sum m(i)=\sum n(i)=0$. Also, we may assume $k>0$.

The first observation is $m(i) \cdot n(i)>0$ for all $i$. Indeed, if $m(i) \cdot n(i)<0$ for some $i$ we may assume $i=s$ without loss of generality and observe $a^{m(s)} b^{n(s)}=$ $b^{n(s)} a^{m(s)} c^{m(s) n(s)}$, so one can find either a shorter word $w^{\prime}$ or larger $k^{\prime}=k-$ $m(s) n(s)$ satisfying $w^{\prime}=c^{k^{\prime}}$, a contradiction.

In the same way, the sign of $m(i)$ cannot be the same as that of $m(i+1)$ for any $i$. Indeed $b^{n(i)} a^{m(i+1)}=c^{-n(i) m(i+1)} a^{m(i+1)} b^{n(i)}$ and as above we get a contradiction.

If one looks for the smallest absolute value of the exponents $m(i)$ and $n(i)$, then (up to cyclic permutation) one can express $w$ as either $x\left(a^{u} \cdot b^{u} \cdot a^{-u}\right) y$ or $x\left(b^{u} \cdot a^{-u} \cdot b^{-u}\right) y$ with $l(w)=l(x)+l(y)+3|u|$. In the first case one has $a^{u} \cdot b^{u}$. $a^{-u}=c^{u^{2}} b^{u}$, so $x \cdot b^{u} \cdot y=c^{k-u^{2}}$. By induction $l(w)-2|u| \geq 2 \sqrt{\left|k-u^{2}\right|}$, so $l(w) \geq 2|u|+2 \sqrt{\left|k-u^{2}\right|} \geq 2 \sqrt{k}$, a contradiction. The second case is similar.

Now $d\left(c^{m}, c^{n}\right)=d\left(c^{m-n}, 1\right) \geq 2 \sqrt{|m-n|}$ by Claim 2 , and $d\left(c^{m}, c^{n}\right) \leq K \sqrt{|m-n|}$ by Claim 1. That means $(\mathbf{Z}, d)$ is bi-Lipschitz equivalent to $(\mathbf{Z}, \sqrt{|m-n|})$. Since $(\mathbf{Z}, \sqrt{|m-n|})$ is quasi-symmetrically equivalent to $(\mathbf{Z},|m-n|)$, we are done.

It is not clear if using methods of 21] one can generalize Proposition 5.1 and prove $\operatorname{asdim}(G)=\operatorname{asdim}_{A N}(G)$ for any virtually nilpotent group $G$.

Question 5.2. Is there a finitely generated group of Assouad-Nagata dimension 1 that is not finitely presented?

\section{REFERENCES}

[1] P. Assouad, Sur la distance de Nagata, C. R. Acad. Sci. Paris Ser. I Math. 294 (1982), no. 1, 31-34. MR651069 (83b:54034)

[2] J. Behrstock, Asymptotic geometry of the mapping class group and Teichmüller space, Geom. Topol. 10 (2006), 1523-1578. MR2255505

[3] J. Behrstock and Y.N. Minsky, Dimension and rank for mapping class groups, preprint ArXiv:math.GT/0512352.

[4] G. Bell and A. Dranishnikov, A Hurewicz-type theorem for asymptotic dimension and applications to geometric group theory, preprint, math.GR/0407431 (2004).

[5] G. Bell and A. Dranishnikov, Asymptotic dimension in Bedlewo, Topology Proceedings (to appear).

[6] N. Brodskiy, J. Dydak, J. Higes, A. Mitra, Nagata-Assouad dimension via Lipschitz extensions, preprint ArXiv:math.MG/0601226, Israel Journal of Math. (to appear).

[7] N. Brodskiy, J. Dydak, M. Levin, A. Mitra, Hurewicz Theorem for Assouad-Nagata dimension, preprint ArXiv:math.MG/0605416, Journal of the London Math. Soc. (to appear).

[8] J. Burillo, Dimension and fundamental groups of asymptotic cones, Journal of the London Math. Soc. 59 (1999), 557-572. MR.1709665 (2000i:20067)

[9] A. Dranishnikov and J. Smith, Asymptotic dimension of discrete groups, Fund. Math. 189 (2006), 27-34. MR2213160(2007h:20041)

[10] A. N. Dranishnikov and J. Smith, On asymptotic Assouad-Nagata dimension, Topology Appl. 154 (2007), 934-952. MR2294641

[11] A. Dranishnikov, M. Zarichnyi, Universal spaces for asymptotic dimension, Topology Appl. 140 (2004), nos. 2-3, 203-225. MR2074917 (2005e:54032)

[12] C. Drutu, Quasi-isometry invariants and asymptotic cones, Int. J. Algebra Comput. 12 (1 and 2) (2002), 99-135. MR1902363 (2003g:20069) 
[13] C. Drutu, M. Sapir, Tree-graded spaces and asymptotic cones of groups, Topology 44 (2005), 959-1058. MR2153979 (2006d:20078)

[14] M. Gromov, Groups of polynomial growth and expanding maps, Publ. Math. IHES 53 (1981), 53-73. MR623534 (83b:53041)

[15] M. Gromov, Asymptotic invariants for infinite groups, in Geometric Group Theory, vol. 2, 1-295, G. Niblo and M. Roller, eds., Cambridge University Press, 1993. MR.1253544 (95m:20041)

[16] J. Heinonen, Lectures on Analysis on Metric Spaces, Universitext, Springer-Verlag, New York, 2001. MR.1800917 (2002c:30028)

[17] U. Lang, T. Schlichenmaier, Nagata dimension, quasisymmetric embeddings, and Lipschitz extensions, IMRN International Mathematics Research Notices (2005), no. 58, 3625-3655. MR2200122 (2006m:53061)

[18] M. Kapovich, Lectures on Geometric Group Theory, preprint (as of September 28, 2005).

[19] J. Nagata, Note on dimension theory of metric spaces, Fund. Math. 45 (1958), 143-181. MR0105081 (21:3827)

[20] P. W. Nowak, On exactness and isoperimetric profiles of discrete groups, J. Funct. Anal. 243 (2007), 323-344. MR2291440

[21] D. V. Osin, Subgroup distortions in nilpotent groups, Comm. Algebra 29 (2001), 5439-5463. MR.1872804 (2002j:20078)

[22] P. Pansu, Croissance des boules et des géodésiques fermées dans les nilvariétés, Ergod. Th. Dynam. Syst. 3 (1983), 415-445. MR741395 (85m:53040)

[23] J. Roe, Lectures on coarse geometry, University Lecture Series, 31, American Mathematical Society, Providence, RI, 2003. MR.2007488 (2004g:53050)

[24] L. van den Dries, A. J. Wilkie, Gromov's theorem on groups of polynomial growth and elementary logic, J. Algebra 89 (1984), 349-374. MR751150(85k:20101)

Department of Mathematics, University of Tennessee, Knoxville, Tennessee 37996

E-mail address: dydak@math.utk.edu

Departamento de Geometría y Topología, Facultad de CC.Matemáticas, Universidad Complutense de Madrid, Madrid, 28040 Spain

E-mail address: josemhiges@yahoo.es 